\title{
Diroximel Fumarate Demonstrates an Improved Gastrointestinal Tolerability Profile Compared with Dimethyl Fumarate in Patients with Relapsing-Remitting Multiple Sclerosis: Results from the Randomized, Double-Blind, Phase III EVOLVE-MS-2 Study
}

\author{
Robert T. Naismith ${ }^{1} \cdot$ Annette Wundes $^{2} \cdot$ Tjalf Ziemssen $^{3} \cdot$ Elzbieta Jasinska $^{4} \cdot$ Mark S. Freedman $^{5}$. \\ Anthony J. Lembo ${ }^{6} \cdot$ Krzysztof Selmaj $^{7,8}$. Ilda Bidollari ${ }^{9} \cdot$ Hailu Chen $^{10}$. Jerome Hanna ${ }^{11}$. \\ Richard Leigh-Pemberton ${ }^{9} \cdot$ Maria Lopez-Bresnahan $^{9} \cdot$ Jennifer Lyons ${ }^{10} \cdot$ Catherine Miller $^{10}$. David Rezendes ${ }^{9}$. \\ Jerry S. Wolinsky ${ }^{12}$ on behalf of The EVOLVE-MS-2 Study Group
}

Published online: 17 January 2020

(c) The Author(s) 2020

\begin{abstract}
Background Diroximel fumarate (DRF) is a novel oral fumarate approved in the USA for relapsing forms of multiple sclerosis. DRF is converted to monomethyl fumarate, the pharmacologically active metabolite of dimethyl fumarate (DMF). DRF $462 \mathrm{mg}$ and DMF $240 \mathrm{mg}$ produce bioequivalent exposure of monomethyl fumarate and are therefore expected to have similar efficacy/safety profiles; the distinct chemical structure of DRF may contribute to its tolerability profile.

Objectives The objective of this study was to compare the gastrointestinal tolerability of DRF and DMF over 5 weeks in patients with relapsing-remitting multiple sclerosis.

Methods EVOLVE-MS-2 was a phase III, randomized, double-blind, head-to-head, 5-week study evaluating the gastrointestinal tolerability of DRF $462 \mathrm{mg}$ vs DMF $240 \mathrm{mg}$, administered twice daily in patients with relapsing-remitting multiple sclerosis, using two self-administered gastrointestinal symptom scales: Individual Gastrointestinal Symptom and Impact Scale (IGISIS) and Global Gastrointestinal Symptom and Impact Scale (GGISIS). The primary endpoint was the number of days with an IGISIS intensity score $\geq 2$ relative to exposure. Other endpoints included the degree of gastrointestinal symptom severity measured by IGISIS/GGISIS and assessment of safety/tolerability.

Results DRF-treated patients experienced a statistically significant reduction (46\%) in the number of days with an IGISIS symptom intensity score $\geq 2$ compared with DMF-treated patients (rate ratio [95\% confidence interval]: 0.54 [0.39-0.75]; $p=0.0003$ ). Lower rates of gastrointestinal adverse events (including diarrhea, nausea, vomiting, and abdominal pain) were observed with DRF than DMF (34.8\% vs 49.0\%). Fewer patients discontinued DRF than DMF because of adverse events (1.6\% vs $5.6 \%)$ and gastrointestinal adverse events ( $0.8 \%$ vs $4.8 \%)$.

Conclusions DRF demonstrated an improved gastrointestinal tolerability profile compared with DMF, with less severe gastrointestinal events and fewer days of self-assessed gastrointestinal symptoms, fewer gastrointestinal adverse events, and lower discontinuation rates because of gastrointestinal adverse events.
\end{abstract}

Clinical Trials Registration ClinicalTrials.gov (NCT03093324).

Maria Lopez-Bresnahan and David Rezendes: Employees of Alkermes Inc. during the time the research and analyses were conducted.

Enhanced Digital Features To view enhanced digital features for this article, go to https://doi.org/10.6084/m9.figshare.11534118.

Electronic supplementary material The online version of this article (https://doi.org/10.1007/s40263-020-00700-0) contains supplementary material, which is available to authorized users.

Extended author information available on the last page of the article

\section{Introduction}

Diroximel fumarate (DRF) is a novel oral fumarate approved in the USA for the treatment of patients with relapsing forms of multiple sclerosis (MS) [1]. DRF undergoes esterase cleavage to monomethyl fumarate, the same pharmacologically active metabolite as the approved drug Tecfidera $^{\circledR}$ (delayed-release dimethyl fumarate [DMF]) [2]. When administered orally at doses of $462 \mathrm{mg}$ and $240 \mathrm{mg}$, respectively, DRF and DMF yield bioequivalent exposure 


\section{Key Points}

EVOLVE-MS-2 was a 5-week head-to-head study evaluating the gastrointestinal tolerability of diroximel fumarate (DRF) vs dimethyl fumarate (DMF) in patients with relapsing-remitting multiple sclerosis.

DRF-treated patients reported less severe gastrointestinal events lasting fewer days compared with patients treated with DMF.

Patients treated with DRF had lower rates of treatment discontinuation due to gastrointestinal adverse events than patients treated with DMF.

of monomethyl fumarate and therefore are expected to have similar efficacy and safety profiles [3]. DMF has demonstrated significant and clinically meaningful efficacy, and a well-tolerated safety profile in clinical trials and real-world studies, totaling $>810,000$ patient-years of exposure [4-8].

Gastrointestinal (GI) adverse events (AEs) such as nausea, vomiting, abdominal pain, and diarrhea are not uncommon with DMF, particularly in the first month of treatment $[4,5$, 9]. In the pivotal DEFINE/CONFIRM trials $(n=1540)$, the incidence of GI AEs was $40 \%$ and led to treatment discontinuation in $4 \%$ of patients $[2,10]$. In a real-world study, up to $88 \%$ of patients treated with DMF reported GI events when self-assessing GI symptoms using eDiaries [9]. Although GI symptom management and mitigation approaches have been developed, DMF treatment discontinuation because of GI AEs still occurs and varies between 5 and $19 \%$ in realworld studies [11, 12].

The distinct chemical structure of DRF is hypothesized to elicit less irritation in the GI tract than DMF through lower production of methanol (a GI-irritating promoiety), and less reactivity with pre-systemic off-target proteins or receptors [13]. Interim findings from the ongoing, multicenter, 2-year, prospective, single-arm, open-label DRF phase III EVOLVE-MS-1 study have demonstrated a low rate $(\sim 31 \%)$ of GI AEs when considered within the context of those reported in separate clinical trials and real-world effectiveness studies of DMF $[9,10,14]$. Notably, $<1 \%$ of patients in the EVOLVE-MS-1 study discontinued DRF treatment because of GI AEs [14]. However, it is challenging to make any conclusions about differences in the GI tolerability profile between DRF and DMF in the absence of head-to-head data.

Herein, we present final study results from the EVOLVEMS-2 study, which was designed to compare GI tolerability of DRF with DMF over 5 weeks in adults with relapsing-remitting MS (RRMS). The EVOLVE-MS-2 study objectives were to (1) evaluate the utility of two GI symptom scales (Individual Gastrointestinal Symptom and Impact Scale [IGISIS] and Global Gastrointestinal Symptom and Impact Scale [GGISIS]); (2) compare GI tolerability of DRF with DMF using these two GI symptom scales; and (3) evaluate the overall safety and tolerability of DRF, including the incidence of GI AEs and number of study withdrawals because of GI AEs in adults with RRMS.

\section{Methods}

\subsection{Study Design}

EVOLVE-MS-2 (NCT03093324) was a 5-week, randomized, double-blind, head-to-head, phase III study designed to evaluate GI tolerability of DRF $462 \mathrm{mg}$ vs DMF $240 \mathrm{mg}$ administered twice daily in patients with RRMS. Patients utilized two eDiary symptom scales to evaluate the duration and severity of GI symptoms on a daily basis: IGISIS and GGISIS. In addition, AEs were collected by investigators at weekly study visits. The study included a $\leq 4$-week screening period, a 5-week double-blind treatment period with two blinded treatment groups, and a 2-week followup period (Fig. 1). The screening period included a 1-week lead-in period, prior to randomization, during which patients completed the two self-administered GI symptom scales daily to test for eDiary compliance and/or underlying baseline GI symptoms.

Block randomization was performed using a block size of 4. Patients were randomized 1:1 into one of the two treatment groups, and all patients received two capsules twice daily for all doses to maintain blinding. Patients received either DRF at the approved dose of $231 \mathrm{mg}$ twice daily (administered as one 231-mg capsule and one placebo capsule twice daily) for week 1 followed by DRF $462 \mathrm{mg}$ twice daily (administered as two 231-mg capsules twice daily) for weeks 2-5 (group 1), or DMF at the approved dose of $120 \mathrm{mg}$ twice daily (administered as one 120-mg capsule and one placebo capsule twice daily) for week 1 followed by DMF $240 \mathrm{mg}$ twice daily (administered as one 240-mg capsule and one placebo capsule twice daily) for weeks 2-5 (group 2). The treatment period was double-blind; DMF capsules were over-encapsulated to create the blinded study drug. Patients were instructed to take the study drug with or without food, but to avoid a high-fat and high-calorie meal (defined as $>1000$ calories and containing $50 \mathrm{~g}$ of fat) to ensure adequate levels of monomethyl fumarate $[2,3]$. No dose reductions were permitted during the study. Symptomatic therapies for tolerability events were permitted and recorded as concomitant medications.

The study utilized an adaptive study design, an approach that allows for planned modifications to ongoing trials (such as changes to trial parameters or statistical procedures) using 
pre-specified interim data analyses, without compromising trial integrity or validity $[15,16]$. Adaptive trial design has been used to re-estimate sample size in instances in which variances of the response variables are unknown, as was the case with the novel endpoints used in the EVOLVEMS-2 study [17]. In this study, it was initially hypothesized that comparing DRF and DMF using the IGISIS intensity scale would detect a difference between the two groups. As there was no previous experience with the IGISIS and GGISIS scales to inform statistical assumptions, a preplanned unblinded analysis of data was conducted after the first 120 patients were randomized (i.e., part A), in which the objectives were to assess the utility of the GI symptom scales; refine the primary endpoint to select the most sensitive measure for detecting a difference between DRF and DMF; and inform the sample size. From this analysis, the IGISIS endpoint was modified from $\geq 3$ to $\geq 2$ as the latter was deemed to be the more sensitive indicator. All patients, investigators, and sites remained blinded to the part A data to preserve the integrity of the trial. After the initial 120 patients, the subsequently randomized patients (i.e., part B) were enrolled, bringing the overall planned population to 500 patients. Patients who completed the 5 -week treatment period were eligible to enroll in the EVOLVE-MS-1 (ClinicalTrials.gov [NCT02634307]) long-term, open-label, DRF safety study [14].

\subsection{Patients}

Eligible patients were aged 18-65 years, had a confirmed diagnosis of RRMS [18], and were neurologically stable with no evidence of relapse in the 30 days prior to screening. Patients were not eligible to participate if they had a history of GI surgery (except appendectomy that occurred $>6$ months prior to screening); clinically significant recurring or active GI symptoms within 3 months of screening or long-term use of medical therapy to treat GI symptoms within 1 month of screening; or two or more IGISIS intensity scores of $\geq 3$ during the 1 -week lead-in period prior to randomization. Patients who had previously received fumarate treatment were also prohibited from study enrollment. The study was approved by central and local ethics committees and was conducted in accordance with the International Council on Harmonisation Guidelines for Good Clinical Practice and the Declaration of Helsinki. All patients provided written informed consent.

\subsection{Study Endpoints}

The primary endpoint was the number of days, relative to exposure, with any IGISIS intensity score $\geq 2$ in the overall study population. Secondary endpoints included the number of days, relative to exposure, with: (1) an IGISIS intensity score $\geq 2$ in part B only; (2) an IGISIS intensity score $\geq 1$ in the overall population; (3) an IGISIS intensity score $\geq 3$ in the overall population; (4) a GGISIS symptom intensity score $\geq 1$ in the overall population; (5) a GGISIS symptom intensity score $\geq 2$ in the overall population; (6) a GGISIS symptom intensity score $\geq 3$ in the overall population; and (7) worst (i.e., highest) IGISIS individual symptom intensity score by week during the 5 -week treatment period in the overall population.

Pre-specified exploratory endpoints included the number of days relative to exposure with an IGISIS intensity score of $\geq 1$ and $\geq 3$, or a GGISIS intensity score of $\geq 1, \geq 2$, or $\geq 3$, in part B only. Investigator-assessed AEs were summarized.

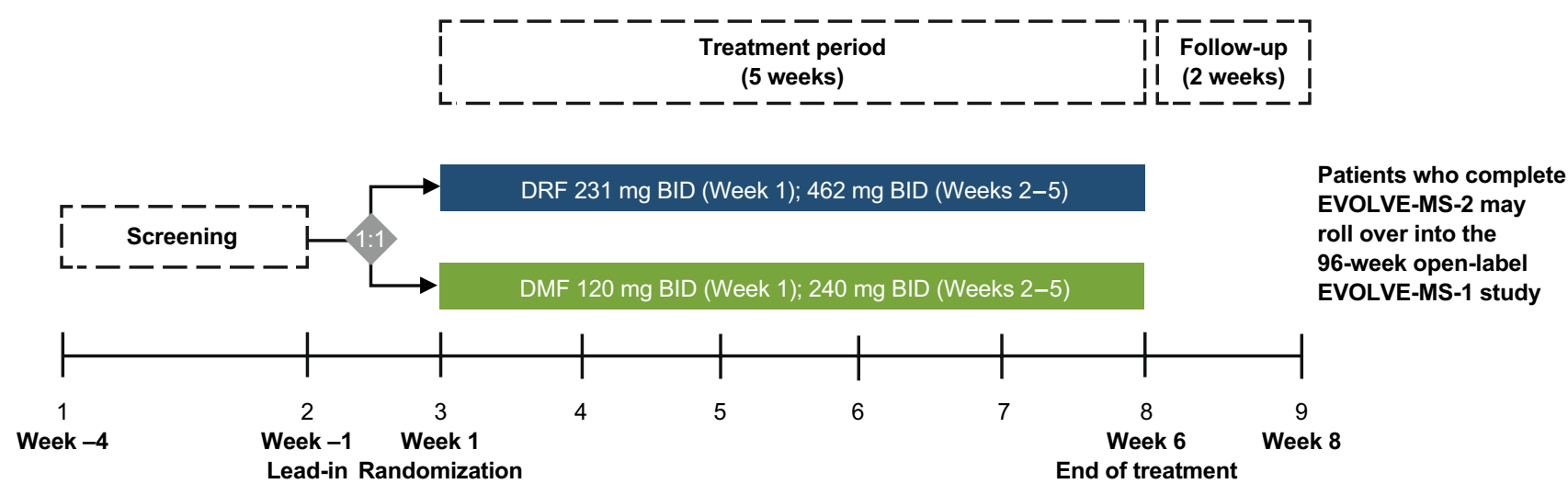

Study visit

Fig. 1 EVOLVE-MS-2 study design. EVOLVE-MS-2 utilized an adaptive study design and was conducted in two parts (A and B). Parts A and B had an identical study design. The first 120 patients randomized were included in part $\mathrm{A}$ and subsequent patients were included in part B. BID twice daily dosing, $D M F$ dimethyl fumarate, $D R F$ diroximel fumarate 


\subsection{Assessments}

\subsubsection{Tolerability Assessments}

Gastrointestinal tolerability was assessed using two novel GI symptom scales, IGISIS and GGISIS. The scales were adapted from the Modified Acute Gastrointestinal Symptom Scale (MAGISS) and the Modified Overall Gastrointestinal Symptom Scale (MOGISS) used in trials with DMF, which have been previously described $[9,19]$. The IGISIS is a questionnaire designed to capture the incidence, intensity, onset, duration, and functional impact of five key individual GI symptoms: nausea, vomiting, upper abdominal pain, lower abdominal pain, and diarrhea (Fig. S1a in the Electronic Supplementary Material [ESM]). In the DMF pivotal DEFINE/CONFIRM trials, these specific GI symptoms were among the most commonly reported AEs and were the most common GI AEs leading to treatment discontinuation $[4,5$, 20]. Patients were instructed to self-administer the IGISIS questionnaire twice per day within $9 \mathrm{~h}$ of taking the study drug, using an eDiary. The patient rated the severity of each symptom on a scale of 0 (did not have) to 10 (extreme); for each symptom, patients also recorded duration and rated interference on daily activities using a 5-point Likert scale (Fig. S1a in the ESM).

The GGISIS is designed to assess the overall intensity of five GI symptoms (nausea, vomiting, upper abdominal pain, lower abdominal pain, and diarrhea) experienced during the previous $24 \mathrm{~h}$, the level of interference and functional impact on work and daily activities, and how bothersome GI symptoms were for patients. To rate the intensity of GI symptoms and assess how bothersome GI symptoms were, patients completed the questionnaire once per day using a scale of 0 (did not have) to 10 (extreme). Patients also recorded the level of interference and impact of GI symptoms on daily activities and work (Fig. S1b in the ESM).

\subsubsection{Safety Assessments}

Safety assessments included AEs (including GI AEs), vital signs, clinical laboratory tests (chemistry, hematology, and urinalysis), and electrocardiogram findings. Adverse events were assessed by the investigator at weekly visits and recorded by severity and relatedness.

\subsection{Analysis Populations and Statistics}

Gastrointestinal tolerability was assessed in all patients who received at least one dose of the study drug and completed at least one post-baseline GI tolerability assessment. Data collected from patients in the overall population were analyzed for the primary endpoint; secondary endpoints assessed data from the overall population, as well as for part B separately.
Based on the pre-planned unblinded analysis of the first 120 patients (part A data), it was assumed that the number of days with an IGISIS intensity score of $\geq 2$ relative to exposure in the treatment period would be 2.0 days for DRF and 3.5 days for DMF. Using a negative binomial regression approach with a two-sided $\alpha$-level of 0.05 , it was estimated that an enrollment size of 500 total patients would provide $\sim 80 \%$ power to detect a $\geq 42 \%$ reduction in the relative rate for DRF vs DMF. The number of days with any IGISIS individual symptom intensity score relative to exposure days was analyzed using a negative binomial regression model with treatment as a factor and adjusted for study parts, region, age, and body mass index. The worst IGISIS individual symptom intensity score during the treatment period was summarized by treatment group and analyzed using an analysis of covariance model with treatment as a factor and adjusted for study parts, region, age, and body mass index. Safety analyses were summarized for all patients who received at least one dose of the study drug.

\section{Results}

\subsection{Patients}

A total of 854 patients underwent screening; 506 patients were randomized and 504 patients received at least one dose of the study drug (Fig. S2 in the ESM). Baseline demographics and disease characteristics were generally well balanced. Approximately $50 \%$ of patients were enrolled from the USA (Table 1). Most patients completed the study (94.8\%, $478 / 504), 97.1 \%$ (464/478) of whom rolled over into the EVOLVE-MS-1 study. The rate of discontinuation was lower for patients treated with DRF compared with DMF (3.2\% vs $7.2 \%$, respectively). This difference in rate of discontinuation was predominantly attributable to the difference in the rate of AEs leading to discontinuation (1.6\% for DRF-treated patients compared with $5.6 \%$ for DMF-treated patients). Other reasons for discontinuation were similar between the two groups (Table 2).

For the analysis of self-assessed GI events, 502 patients were eligible for inclusion in the analysis; 253 were assigned to DRF and 249 were assigned to DMF. For the IGISIS analyses, mean (standard deviation [SD]) exposure days were comparable for the two groups: 35.2 (4.2) days for DRFtreated patients and 34.2 (5.9) days for DMF-treated patients. When analyzing the part B population separately, 194 DRFtreated and 191 DMF-treated patients were included. Mean (SD) exposure in the part B population was similar to that of the overall population: 35.2 (3.8) days for DRF and 33.7 (6.3) days for DMF. For the GGISIS analyses, mean (SD) exposure days with at least one diary available for analysis were lower than that observed with IGISIS, yet comparable 
between the two groups: 33.3 (4.8) days for DRF and 32.6 (5.6) days for DMF.

\subsection{Patient Self-Assessed Gastrointestinal Tolerability}

The number of days with an IGISIS intensity score of $\geq 2$ relative to exposure was statistically significantly lower with DRF compared with DMF. The adjusted mean (95\% confidence interval $[\mathrm{CI}]$ ) number of days with a patient-assessed event was $1.4(1.1-1.9)$ days with DRF and $2.6(2.0-3.3)$ days with DMF. The adjusted rate ratio $(95 \% \mathrm{CI})$ was 0.54 $(0.39-0.75)$, representing a $46 \%$ reduction $(p=0.0003$; Fig. 2). A sensitivity analysis was performed to include only diaries completed as instructed (within 2-9 h of dosing). The mean (SD) number of evaluable days with diaries completed as instructed were similar for the two groups: 25.8 (11.8) for DRF and 26.1 (11.0) for DMF. Among diaries completed as instructed, the adjusted mean ( $95 \% \mathrm{CI}$ ) number of days with an IGISIS score of $\geq 2$ relative to exposure was $1.0(0.8-1.3)$ for DRF and $2.1(1.6-2.9)$ for DMF, resulting in an adjusted rate ratio $(95 \% \mathrm{CI})$ of $0.49(0.34-0.70)$; this represented a $51 \%$ reduction $(p<0.0001)$.

When symptom intensity and frequency was assessed using a GGISIS intensity score of $\geq 2$, there were fewer days of events with DRF compared with DMF, although the difference was not statistically significant. Patients treated with DRF reported $1.1(0.8-1.5)$ adjusted mean $(95 \% \mathrm{CI})$ days with a GGISIS score of $\geq 2$ compared with 1.6 (1.1-2.2) days for DMF-treated patients in the overall population. The adjusted rate ratio $(95 \% \mathrm{CI})$ was $0.67(0.43-1.05 ; p=0.082$; Fig. 2).

In a comparison using an IGISIS intensity score of $\geq 1$, the adjusted mean $(95 \% \mathrm{CI})$ number of days relative to exposure was significantly lower with DRF (3.0 [2.5-3.7]) compared with DMF (4.1 [3.4-5.0]). The rate ratio (95\% CI) was $0.71(0.55-0.92)$, representing a $29 \%$ reduction $(p=0.009$; Fig. 2). The number of days relative to exposure with an IGISIS intensity score of $\geq 3$ was $44 \%$ lower with DRF than DMF (rate ratio [95\% CI]: 0.56 [0.36-0.86]; $p=0.009)$. Similarly, there were fewer days with a GGISIS intensity score of $\geq 1$ (rate ratio [95\% CI]: 0.70 [0.50-0.98]) and $\geq 3(0.74$ [0.43-1.28]) with DRF than DMF over the study period (Fig. 2).

In an analysis of data collected from part B only, the number of days with an IGISIS intensity score of $\geq 2$ relative to exposure was statistically significantly lower with DRF compared with DMF. The adjusted mean $(95 \% \mathrm{CI})$ number of days with a patient-assessed event for DRF-treated patients was $1.2(0.9-1.5)$ days compared with $2.1(1.7-2.8)$ days for DMF-treated patients. The adjusted rate ratio $(95 \% \mathrm{CI})$ was $0.52(0.36-0.76)$, representing a $48 \%$ reduction $(p=0.0007$; Fig. 3). Reductions consistent with those observed in the
Table 1 Baseline demographics and disease characteristics in EVOLVE-MS-2

\begin{tabular}{lcc}
\hline & DRF & DMF \\
& $n=253$ & $n=251$ \\
\hline Mean (SD) age, years & $43.7(10.96)$ & $43.7(9.90)$ \\
Female, $n(\%)$ & $177(70.0)$ & $190(75.7)$ \\
Race, $n(\%)$ & & \\
White & $232(91.7)$ & $227(90.4)$ \\
Black or African American & $20(7.9)$ & $20(8.0)$ \\
Other & $1(0.4)$ & $4(1.6)$ \\
Mean (SD) weight, kg & $78.0(18.7)$ & $78.2(19.6)$ \\
Mean (SD) BMI, kg/m ${ }^{2}$ & $27.2(5.9)$ & $27.5(6.1)$ \\
US region, $n$ (\%) & $135(53.4)$ & $143(57.0)$ \\
Prior DMT, $n(\%)$ & & \\
0 & $84(33.2)$ & $85(33.9)$ \\
1 & $73(28.9)$ & $72(28.7)$ \\
2 & $60(23.7)$ & $43(17.1)$ \\
$\geq 3$ & $36(14.2)$ & $51(20.3)$ \\
Mean (SD) time since diagnosis, years & $7.4(7.80)$ & $7.9(7.37)$ \\
Mean (SD) time since first symptom, & $9.6(8.96)$ & $10.1(8.55)$ \\
years & & \\
Mean (SD) no. of relapses in previous & $0.6(0.72)$ & $0.6(0.72)$ \\
year & & \\
Mean (SD) EDSS score & $2.70(1.407)$ & $2.72(1.380)$ \\
Mean (SD) no. of Gd +lesions ${ }^{\mathrm{a}}$ & $0.9(2.22)$ & $1.1(2.76)$ \\
Patients with 0 Gd + lesions, $n(\%)$ & $180(71.1)$ & $175(69.7)$ \\
\hline
\end{tabular}

$B M I$ body mass index, $D M F$ dimethyl fumarate, $D M T$ disease-modifying therapy, $D R F$ diroximel fumarate, EDSS Expanded Disability Status Scale, $G d+$ gadolinium-enhancing, $S D$ standard deviation

${ }^{\mathrm{a} D R F}, n=251$; DMF, $n=251$

overall population were also noted when comparing DRF and DMF using IGISIS intensity scores of $\geq 1 \quad(p=0.01)$ and $\geq 3(p=0.012)$ and GGISIS intensity scores of $\geq 1$ $(p=0.028), \geq 2$, and $\geq 3$ in the part B-only population (Fig. $\mathrm{S} 3$ in the ESM).

Symptom severity was assessed using data collected from the IGISIS eDiary. Over the course of the 5-week treatment period, patients recorded an overall least squares (LS) mean (standard error [SE]) worst IGISIS symptom intensity score of $2.0(0.2)$ if treated with DRF and $2.4(0.2)$ if treated with DMF ( $p=0.069$; Fig. 4). During the 1-week titration period, LS mean (SE) worst symptom score was $1.0(0.1)$ for DRF vs $0.8(0.1)$ for DMF. However, following the titration period, LS mean (SE) worst symptom intensity score peaked after the first week of the full-dose study drug for DMFtreated patients (DMF week 3, 1.4 [0.1]), before declining by the end of treatment (DMF week 5, 0.6 [0.1]). In contrast, DRF-treated patients experienced a gradual decline in LS mean (SE) worst symptom score over the course of treatment (DRF week 3, 0.9 [0.1]; week 5, 0.5 [0.1]). Statistically 
Table 2 Disposition of patients

\begin{tabular}{lll}
\hline Patients, $n(\%)$ & \multicolumn{2}{l}{ Treatment group } \\
\cline { 2 - 3 } & DRF & DMF \\
\hline Randomized & $254(100)$ & $252(100)$ \\
Received at least one dose of the study drug & $253(99.6)$ & $251(99.6)$ \\
Completed the treatment period & $245(96.8)$ & $233(92.8)$ \\
Completed the study & $245(96.8)$ & $233(92.8)$ \\
Rolled over to EVOLVE-MS-1 & $239(94.5)$ & $225(89.6)$ \\
Discontinued during the study period & $8(3.2)$ & $18(7.2)$ \\
Adverse events & $4(1.6)$ & $15(6.0)$ \\
Lost to follow-up & $1(0.4)$ & 0 \\
Protocol deviation & $1(0.4)$ & $1(0.4)$ \\
Withdrawal by patient & $2(0.8)$ & $2(0.8)$ \\
Lack of efficacy & 0 & 0 \\
Physician decision & 0 & 0 \\
Other & 0 & 0 \\
\hline
\end{tabular}

$D M F$ dimethyl fumarate, $D R F$ diroximel fumarate

${ }^{a}$ End of study included completion of the treatment period in patients rolling over into the EVOLVE-MS-1 trial, and completion of the treatment period and the 2-week follow-up period in patients who did not roll over into the EVOLVE-MS-1 trial

significant differences with DRF vs DMF were observed in week 3 (DRF: 0.9 [0.1] vs DMF: 1.4 [0.1], $p=0.002$ ) and week 4 (DRF: 0.6 [0.1] vs DMF: 1.0 [0.1], $p=0.004$ ). The IGISIS worst symptom intensity scores were lower with DRF than DMF for events associated with the upper GI tract (with statistically significant reductions observed for nausea, vomiting, upper abdominal pain) but similar for events associated with the lower GI tract (diarrhea, lower abdominal pain; Table 3).

Fewer patients treated with DRF categorized their GI events as interfering "quite a bit" or "extremely" with the ability to accomplish daily activities when assessed using both IGISIS and GGISIS scales, compared with patients treated with DMF: nausea (2.4\% vs $6.8 \%)$, vomiting (1.2\% vs $5.6 \%)$, upper abdominal pain $(1.2 \%$ vs $6.8 \%)$, lower abdominal pain ( $1.2 \%$ vs $3.2 \%)$, diarrhea (3.6\% vs $6.4 \%)$, and all GI events (GGISIS, $7.9 \%$ vs $10.8 \%$; Table S1 in the ESM). In addition, a higher percentage of patients treated with DRF assessed the impact of their GI events as "not at all" affecting work productivity $(47.8 \%, 121 / 253)$ compared with DMF $(40.6 \%, 101 / 249)$, and for those reporting employment and missed hours at work because of GI events (DRF, 20/133; DMF, 26/133) the mean (SD) greatest number of hours missed was lower for patients treated with DRF than DMF: 4.3 (3.7) and 5.5 (4.8), respectively.

\subsection{Safety}

Overall, AEs were reported in $81 \%$ (408/504) of patients (DRF, 78.3\%; DMF, 83.7\%; Table 4). Most AEs were mild to moderate in severity (DRF, 97.5\% [193/198]; DMF, 93.3\% [196/210]; Table 4). The overall rate of serious AEs was low (1.4\%; four patients with DRF and three patients with DMF); none were related to the study drug. No deaths

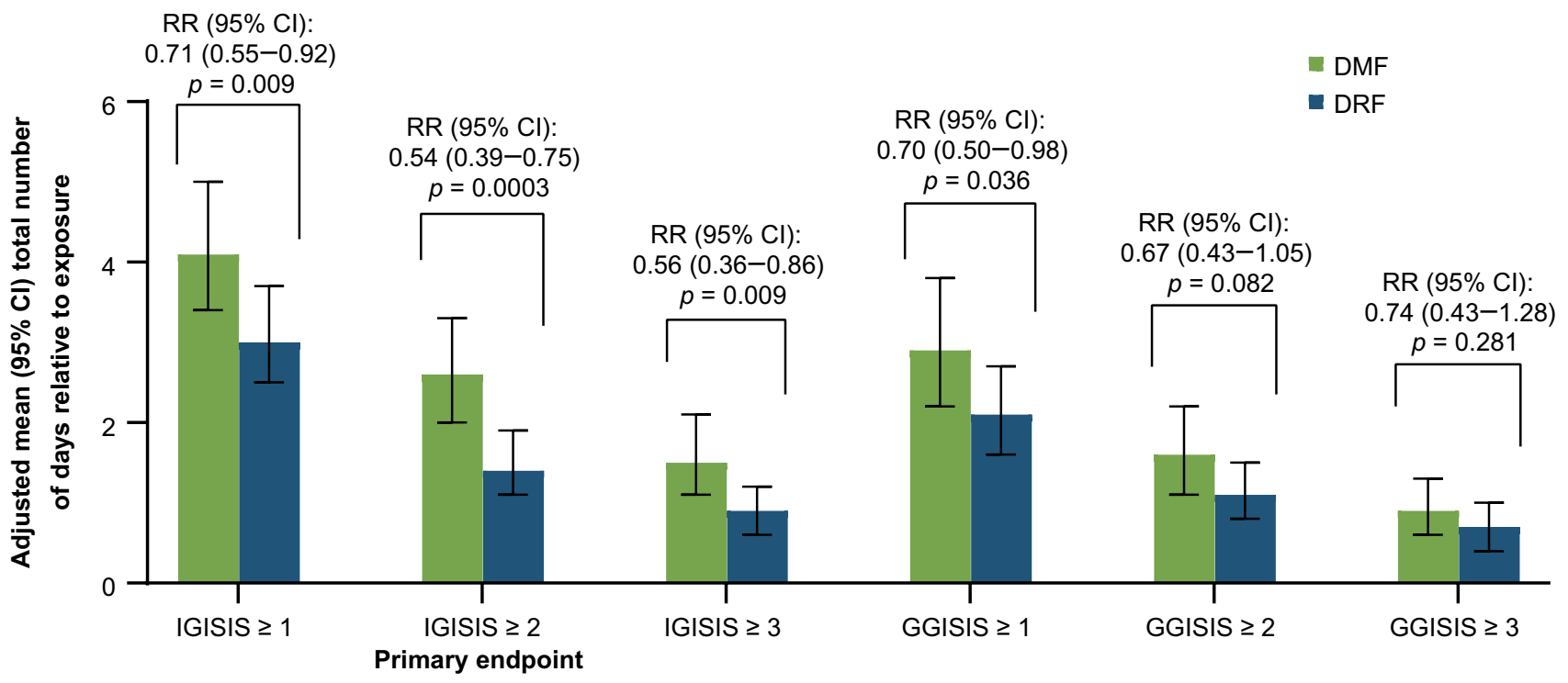

Gastrointestinal symptom intensity score

Fig. 2 Primary and secondary endpoints in the overall population. For the overall population: diroximel fumarate (DRF), $n=253$; dimethyl fumarate (DMF), $n=249$. $C I$ confidence interval, GGISIS Global
Gastrointestinal Symptom and Impact Scale, IGISIS Individual Gastrointestinal Symptom and Impact Scale, $R R$ rate ratio 
were reported. Gastrointestinal AEs were among the most frequently reported; $34.8 \%$ in the DRF treatment group and $49.0 \%$ in the DMF treatment group. In particular, GI AEs associated with an upper GI location (upper abdominal pain, nausea, vomiting) appeared to be reported with less frequency in patients treated with DRF compared with DMF (Table 5). Overall, 19.3\% (17/88) of patients in the DRF group and $30.6 \%(37 / 121)$ of patients in the DMF group used concomitant medications to treat GI-related AEs during the treatment period. Two patients in the DMF group experienced a GI AE that was not considered tolerability related (toothache, $n=1$; dry mouth, $n=1$ ) and were excluded from the analysis. Flushing was reported in $36.7 \%$ of patients overall (DRF, 32.8\%; DMF, 40.6\%).

The incidence of AEs leading to treatment discontinuation was lower for patients treated with DRF (1.6\%) compared with DMF (5.6\%). Gastrointestinal AEs led to discontinuation in $0.8 \%$ of patients treated with DRF and $4.8 \%$ of patients treated with DMF. No patients in either treatment group discontinued because of lack of efficacy.

\section{Discussion}

Results from the EVOLVE-MS-2 study demonstrate that DRF has an improved GI tolerability profile compared with DMF. Tolerability is an important factor for drug adherence and achievement of maximal efficacy, particularly for medications used for long-term management of chronic diseases such as MS [21]. This randomized double-blind study was specifically designed to assess the duration and severity of key GI events: nausea, vomiting, diarrhea, and upper and lower abdominal pain.

EVOLVE-MS-2 is the first study to directly compare the GI tolerability profiles of two MS treatments. Patients treated with DRF reported significantly fewer days with GI events, with an IGISIS severity score of $\geq 2$ over a 5 -week period, than patients treated with DMF. This finding, which favored DRF, was consistently observed across comparisons using different severity score thresholds on both IGISIS and GGISIS. The observed differences in days with GI events and severity of GI events as assessed by the patient were supported by lower rates of investigator-assessed GI AEs and GI AEs leading to treatment discontinuation in DRF-treated patients. In addition, patients treated with DRF tended to report that GI events were less likely to interfere with their daily activities, less bothersome, and less likely to impact work productivity and absenteeism. Although the absolute difference in number of days with GI events with DRF and DMF as measured by IGISIS was small, the improvements observed with DRF support clinically meaningful and relevant outcomes such as fewer treatment discontinuations, less patient-reported interference of GI symptoms, and fewer effects on work productivity. The timing of treatment benefit is also important. Most $(91.7 \%$; 11/12) DMF treatment discontinuations in EVOLVE-MS-2 occurred by week 3. Therefore, although worst GI symptom severity scores with DRF and DMF tend to be similar by week 5 (Fig. 4), the early differences observed by week 3 appear to provide meaningful patient benefit.

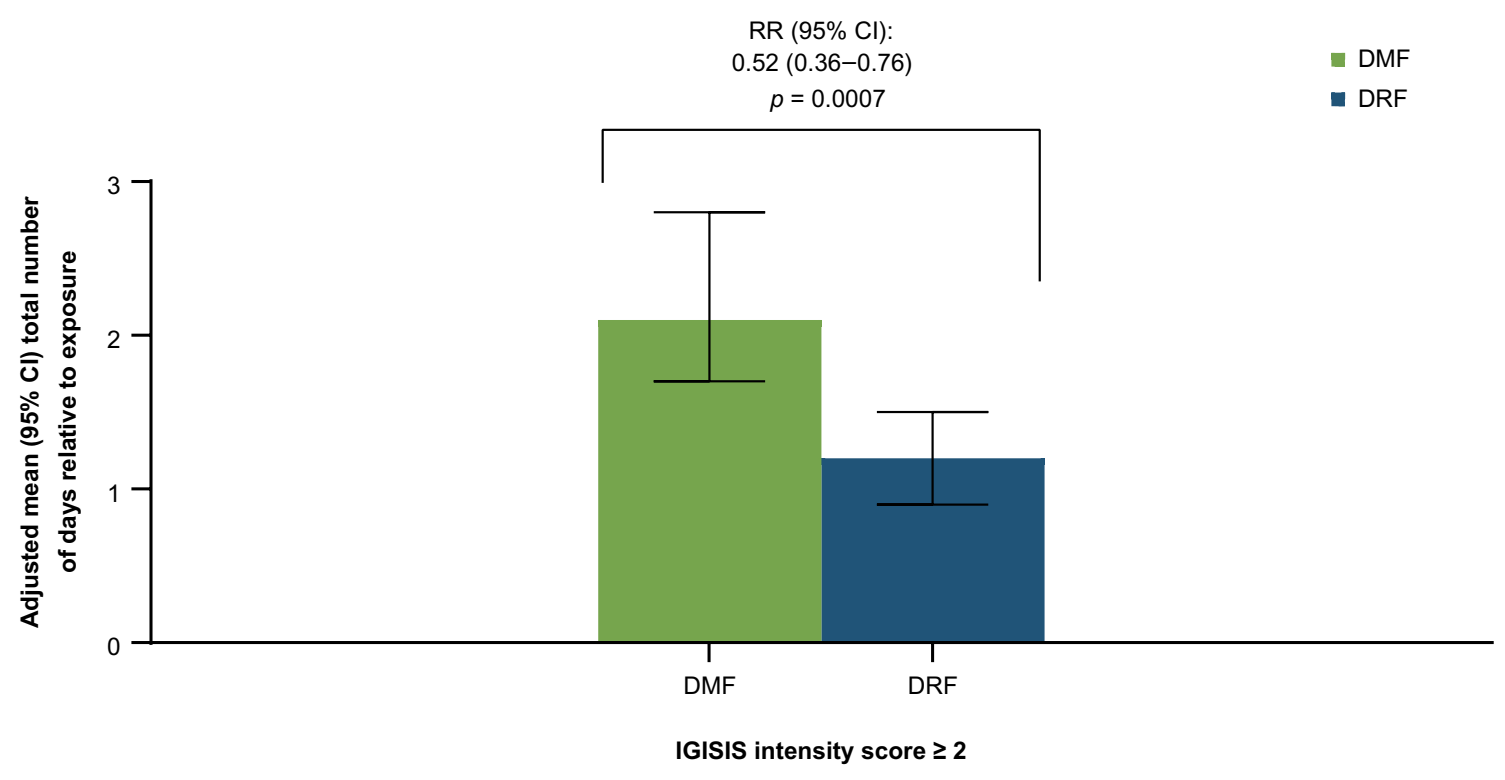

Fig. 3 Number of days relative to exposure with an Individual Gastrointestinal Symptom and Impact Scale (IGISIS) intensity score $\geq 2$ for diroximel fumarate (DRF) vs dimethyl fumarate (DMF) in patients enrolled after the pre-planned analysis of the first 120 patients (part B only). For part B only: DRF, $n=194$; DMF, $n=191$. $C I$ confidence interval, $R R$ rate ratio 


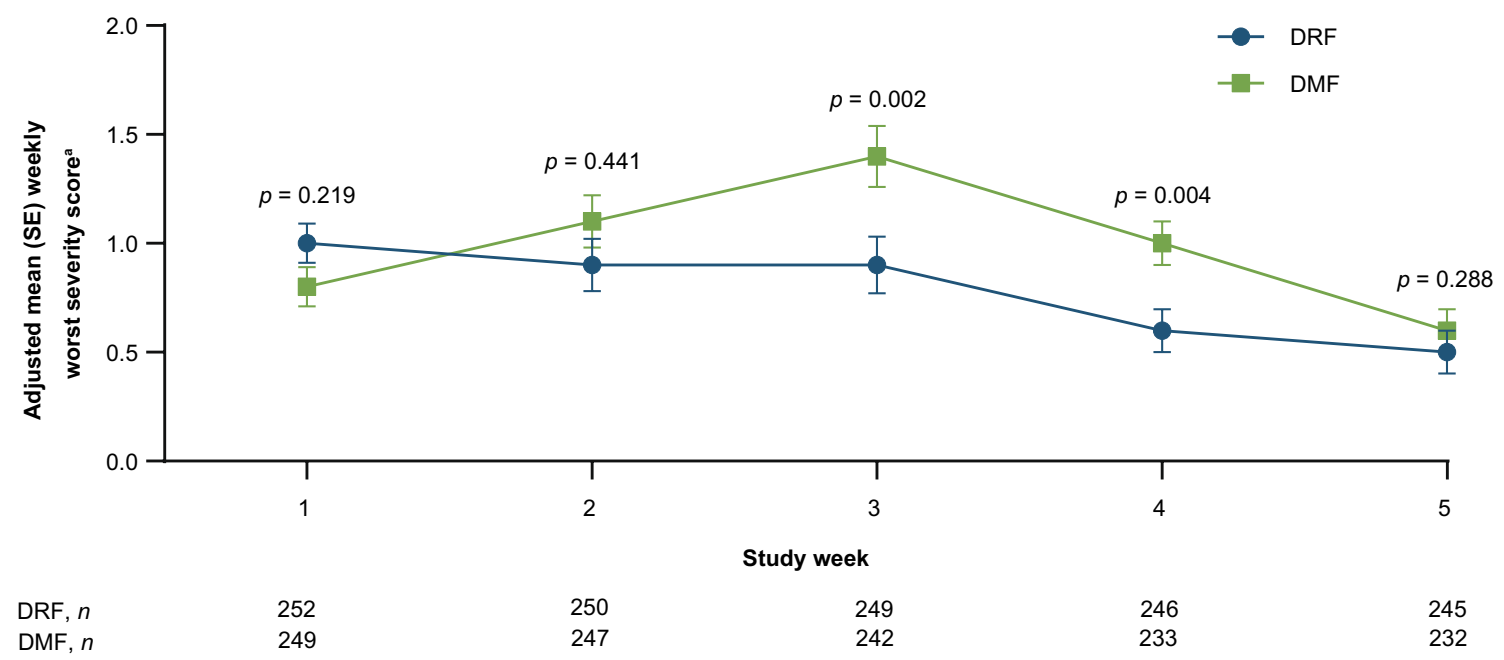

Fig. 4 Mean worst severity score for gastrointestinal events (Individual Gastrointestinal Symptom and Impact Scale [IGISIS]) by weekly interval in the overall population. $D M F$ dimethyl fumarate,

Table 3 Individual Gastrointestinal Symptom and Impact Scale (IGISIS) worst individual symptom intensity score by gastrointestinal (GI) location reported during the 5-week treatment period

\begin{tabular}{lll}
\hline $\begin{array}{l}\text { IGISIS worst individual symptom } \\
\text { intensity score }\end{array}$ & $\begin{array}{l}\text { DRF } \\
n=253\end{array}$ & $\begin{array}{l}\text { DMF } \\
n=249\end{array}$ \\
\hline $\begin{array}{l}\text { Upper GI events } \\
\text { Upper abdominal pain }\end{array}$ & & \\
$\quad$ LS mean (SE) & & \\
$\quad P$ value & $0.8(0.1)$ & \\
Nausea & 0.001 & $1.3(0.1)$ \\
$\quad$ LS mean $(\mathrm{SE})^{\mathrm{a}}$ & & \\
$P$ value & $1.0(0.1)$ & \\
Vomiting & 0.043 & $0.7(0.1)$ \\
$\quad$ LS mean (SE) & \\
$P$ value & $0.2(0.1)$ & \\
Lower GI events & $<0.001$ & $1.0(0.1)$ \\
Lower abdominal pain & & \\
$\quad$ LS mean $(\mathrm{SE})^{\mathrm{a}}$ & & $1.4(0.2)$ \\
$P$ value & $0.9(0.1)$ & \\
Diarrhea & 0.403 & \\
$\quad$ LS mean $(\mathrm{SE})^{\mathrm{a}}$ & $1.1(0.1)$ & \\
$P$ value & 0.261 & \\
\hline
\end{tabular}

$D M F$ dimethyl fumarate, $D R F$ diroximel fumarate, $L S$ least squares, $S E$ standard error

${ }^{a}$ LS mean symptom intensity score was analyzed using an analysis of covariance model adjusted for study parts, region (USA vs non-USA), age, and body mass index

For DRF, fewer upper GI events (nausea, vomiting, upper abdominal pain) were reported when assessed by patients and investigators, which is consistent with the hypothesis that DRF may potentially elicit less localized GI irritation
$D R F$ diroximel fumarate, $S E$ standard error. ${ }^{a}$ Analysis of covariance model; factors include study parts, region (USA and non-USA), age, and body mass index

Table 4 On-treatment safety summary

\begin{tabular}{llc}
\hline TEAE, $n(\%)$ & $\begin{array}{l}\text { DRF } \\
n=253\end{array}$ & $\begin{array}{l}\text { DMF } \\
n=251\end{array}$ \\
\hline Any TEAE & $198(78.3)$ & $210(83.7)$ \\
Mild & $125(49.4)$ & $121(48.2)$ \\
Moderate & $68(26.9)$ & $75(29.9)$ \\
Severe & $5(2.0)$ & $14(5.6)$ \\
Serious AE & $4(1.6)$ & $3(1.2)$ \\
AE leading to discontinuation ${ }^{\mathrm{b}}$ & $4(1.6)$ & $14(5.6)^{\mathrm{c}}$ \\
GI AE leading to discontinuation $^{\mathrm{a}}$ & $2(0.8)$ & $12(4.8)$ \\
Upper abdominal pain $_{\text {Diarrhea }}$ & 0 & $5(2)$ \\
Abdominal pain & $1(0.4)$ & $3(1.2)$ \\
Vomiting $_{\text {Abdominal distension }}$ & 0 & $3(1.2)$ \\
GI pain & $1(0.4)$ & $2(0.8)$ \\
Nausea & 0 & $1(0.4)$ \\
\hline
\end{tabular}

$A E$ adverse event, $D M F$ dimethyl fumarate, $D R F$ diroximel fumarate, GI gastrointestinal, TEAE treatment-emergent adverse event

${ }^{a}$ Serious AEs in DRF-treated patients included: multiple sclerosis relapse, $n=2$; multiple sclerosis relapse and suicide attempt, $n=1$; atrial fibrillation, $n=1$. Serious AEs in DMF-treated patients included: multiple sclerosis relapse, $n=2$; cholecystitis, $n=1$

${ }^{\mathrm{b}}$ AEs leading to DRF treatment discontinuation were GI AEs $(n=2$, listed in table), dermatitis allergic $(n=1)$, and suicide attempt $(n=1)$. AEs leading to DMF treatment discontinuation were GI AEs $(n=12$, listed in table), depression $(n=1)$, and urticaria $(n=1)$

${ }^{c}$ One patient in the DMF arm reported an AE after the last study-dose date (post-treatment period, during the follow-up period) that led to discontinuation from the study. This patient is captured as having an AE leading to discontinuation from the study (Table 2) but not as having an $\mathrm{AE}$ leading to discontinuation during the treatment period 
compared with DMF, owing to its distinct chemical structure [13]. The potential impact would likely be most evident in the upper GI tract, given that both DRF and DMF are formulated to be released from their capsules and microspheres upon traversing from the stomach into the small intestine; this region of the GI tract would be exposed to the highest concentrations of DRF and DMF, allowing for greater differentiation of their tolerability effects. This was reflected in the worst symptom intensity scores (IGISIS), which show statistically significant differences in favor of DRF for upper GI tract symptoms.

The IGISIS and GGISIS scales were utilized for this study to assess GI symptoms given that evaluation of GI events is not a typical assessment for the management of MS, and no validated scales are currently available to measure such outcomes. As there was no previous experience with the IGISIS and GGISIS scales to inform statistical assumptions, an adaptive trial design was used to enable the selection of a sensitive primary endpoint and inform statistical assumptions for the overall study (see Methods). To mitigate the possibility of a type I error, an analysis to validate the primary endpoint only in patients enrolled after the unblinding (i.e., part B) was included. Results from the part B-only analysis were aligned with that for the overall population on the primary endpoint $46 \%$ reduction in the total population; $48 \%$ reduction in the part $\mathrm{B}$ cohort) as well as secondary endpoints.

Interestingly, the rates of investigator-assessed GI AEs and discontinuations because of GI AEs reported for DRFand DMF-treated patients were consistent with rates in the ongoing EVOLVE-MS-1 study, as well as with rates in the pivotal DEFINE and CONFIRM studies, though the trials cannot be directly compared $[4,5,14]$. The incidence of flushing, though numerically lower with DRF than DMF in this study, was generally consistent with rates reported in phase III studies with DRF and DMF [4, 5, 14]. However, as the focus of EVOLVE-MS-2 was GI tolerability, we did not evaluate and cannot assess the impact of flushing on patients in the study. There were no unexpected or new safety events, including lymphopenia, reported for patients taking either DRF or DMF, and the overall safety profile was consistent with the known safety profile for each therapy, including interim findings from the ongoing open-label EVOLVE-MS-1 study [14].

There were limitations of the study. There is potential for bias toward over-reporting when patients self-assess GI events in a study designed to measure GI tolerability using eDiaries three times per day. In this setting, patients were

Table 5 Treatment-emergent adverse events (TEAEs) experienced in $\geq 5 \%$ of patients (in any group)

\begin{tabular}{|c|c|c|}
\hline \multirow[t]{2}{*}{ System organ class preferred term, $n(\%)$} & \multicolumn{2}{|c|}{ Treatment groups } \\
\hline & $\begin{array}{l}\text { DRF } \\
(n=253)\end{array}$ & $\begin{array}{l}\text { DMF } \\
(n=251)\end{array}$ \\
\hline Any TEAE & $198(78.3)$ & $210(83.7)$ \\
\hline Gastrointestinal disorders & $88(34.8)$ & $123(49.0)$ \\
\hline Diarrhea & $39(15.4)$ & $56(22.3)$ \\
\hline Nausea & $37(14.6)$ & $52(20.7)$ \\
\hline Upper abdominal pain & $17(6.7)$ & $39(15.5)$ \\
\hline Abdominal pain & $16(6.3)$ & $24(9.6)$ \\
\hline Lower abdominal pain & $15(5.9)$ & $17(6.8)$ \\
\hline Vomiting & $9(3.6)$ & $22(8.8)$ \\
\hline General disorders and administration site conditions & $16(6.3)$ & $30(12.0)$ \\
\hline Fatigue & $6(2.4)$ & $13(5.2)$ \\
\hline Infections and infestations & $43(17.0)$ & $35(13.9)$ \\
\hline Nasopharyngitis & $15(5.9)$ & $11(4.4)$ \\
\hline Investigations & $27(10.7)$ & $24(9.6)$ \\
\hline Alanine aminotransferase increased & $14(5.5)$ & $9(3.6)$ \\
\hline Nervous system disorders & $37(14.6)$ & $34(13.5)$ \\
\hline Headache & $10(4.0)$ & $14(5.6)$ \\
\hline Skin and subcutaneous tissue disorders & $49(19.4)$ & $58(23.1)$ \\
\hline Erythema & $20(7.9)$ & $21(8.4)$ \\
\hline Pruritus & $18(7.1)$ & $18(7.2)$ \\
\hline Vascular disorders & $88(34.8)$ & $107(42.6)$ \\
\hline Flushing & $83(32.8)$ & $102(40.6)$ \\
\hline
\end{tabular}

$D M F$ dimethyl fumarate, $D R F$ diroximel fumarate 
aware via their informed consent that they could potentially receive an investigational product that would reduce GI events, which may have conversely resulted in less reporting overall. Owing to the prospective randomized blinded nature of the study, no bias would have been introduced, although it may have artificially lowered the overall magnitude of difference detected between both arms. The IGISIS and GGISIS scales capture five GI symptoms (nausea, vomiting, upper abdominal pain, lower abdominal pain, and diarrhea) that were most commonly reported in the DEFINE and CONFIRM trials of DMF. However, patients in EVOLVE-MS-2 did experience GI AEs beyond the five included in the IGISIS/GGISIS assessments. Although the scales do not capture every GI AE, the events that are not included occur at low rates (incidence $\leq 2 \%$ ). Additionally, the short duration of treatment ( 5 weeks) limits the ability to determine the time of resolution of AEs that were ongoing at the study end, and prohibits the assessment of AEs that occur with a latency. Hence, it does not fully describe the AE profile of DRF and DMF, although this was not the intent of the study. Despite this limited follow-up, the AE profile was consistent with the known profiles for both DRF and DMF, and the high percentage of patients rolling over into the EVOLVE-MS-1 study will allow for the evaluation of the DRF AE profile for up to 2 years on treatment.

\section{Conclusions}

In this 5-week head-to-head study evaluating the GI tolerability of DRF vs DMF in patients with RRMS, DRF-treated patients assessed their GI events as less severe and lasting fewer days when compared with patients treated with DMF. Importantly, rates of GI AEs and rates of discontinuation due to GI AEs were lower for patients treated with DRF. Taken together, these findings indicate that DRF has an improved GI tolerability profile compared with DMF, which may lead to better long-term adherence and persistence to therapy.

\footnotetext{
Acknowledgements The authors thank Jordan Messer, PharmD, Narinder Nangia, PhD, Yue Cui, PhD, and Mary-Kathryn Canning, MBA for their contributions to this manuscript. Biogen provided funding for medical writing support in the development of this manuscript. Susan Chow, $\mathrm{PhD}$, from Excel Scientific Solutions wrote sections of the first draft of the manuscript based on input from authors, and Miranda Dixon from Excel Scientific Solutions copyedited and styled the manuscript per journal requirements. The authors had full editorial control of the manuscript and provided their final approval of all content.
}

Author Contributions RTN, AW, TZ, EJ, AJL, KS, and DR participated in the collection of data. RTN, IB, RL-P, and JSW participated in the study design and statistical analysis plan. RTN, AW, TZ, EJ, MSF, AJL, KS, IB, HC, JH, RL-P, ML-B, JL, CM, DR, and JSW participated in the analysis and interpretation of data. HC performed the statistical analysis. RTN, AW, TZ, EJ, MSF, AJL, IB, JH, RL-P,
ML-B, JL, CM, DR, and JSW participated in drafting and critically revising the manuscript.

Data Availability Statement EVOLVE-MS-2 was registered with ClinicalTrials.gov (NCT03093324). Study data will be shared in accordance with applicable regulations and laws.

\section{Compliance with Ethical Standards}

Funding This study was funded by Alkermes Inc. (Waltham, MA, USA) and Biogen (Cambridge, MA, USA). The open access fee was funded by Biogen.

Conflict of interest Robert T. Naismith received compensation as an advisor, consultant, or speaker for Alexion, Alkermes, Biogen, Celgene, EMD Serono, Genentech, Novartis, Sanofi Genzyme, TG Therapeutics, and Viela Bio. Annette Wundes received research support from AbbVie, Alkermes, and Biogen, and compensation as an advisor from Biogen. Tiaf Ziemssen received fees for participation in scientific advisory boards from Bayer, Biogen Idec, Sanofi Genzyme, Merck Serono, Novartis, Synthon, and Teva; speaker honorarium from Almirall, Bayer, Biogen, GlaxoSmithKline, Merck Sharp \& Dohme, Novartis, Sanofi Genzyme, and Teva; and research support from Bayer, Biogen, Novartis, Sanofi Genzyme, and Teva. Elzbieta Jasinska received fees for participation in scientific advisory boards from Biogen and speaker honoraria from Adamed, Allergan, Hoffman-La Roche, Krka, Novartis, Polpharma, and Teva. Mark S. Freedman received research or educational grants from Genzyme Canada; honoraria or consultation fees from Actelion, Bayer, Biogen, Celgene, Chugai, EMD Serono Canada, Hoffman-La Roche, Merck Serono, Novartis, PendoPharm, Sanofi-Aventis, and Sanofi Genzyme; participated as a member in a company advisory board, board of directors, or other similar group for Actelion, Bayer, Biogen, Clene, Hoffman-La Roche, MedDay, Merck Serono, Novartis, and Sanofi-Aventis; and served on a companysponsored speaker's bureau for Sanofi Genzyme. Anthony J. Lembo received fees for participation in scientific advisory boards from Bayer, Biogen Idec, Ironwood, Salix, Shire, and Takeda. Krzysztof Selmaj received consulting fees from Genzyme, Novartis, Ono, Roche, Synthon, and Teva, and speaker fees from Biogen. Ilda Bidollari, Richard Leigh-Pemberton, Maria Lopez-Bresnahan, and David Rezendes are employees of and hold stock/stock options in Alkermes. Hailu Chen, Jerome Hanna, Jennifer Lyons, and Catherine Miller are employees of and hold stock/stock options in Biogen. Jerry S. Wolinsky received compensation for consulting, scientific advisory boards, or other activities with AbbVie, Acorda Therapeutics, Alkermes, Brain Cell Therapeutics, Celgene (Exp 11 2019), Clene Nanomedicine, EMD Serono, Forward Pharma, GeNeuro, GW Pharma, MedDay Pharmaceuticals, NervGen Pharma Corp., Novartis, Otsuka, PTC Therapeutics, Roche/ Genentech, and Sanofi Genzyme; compensation for CME activities with AcademicCME, PlatformQ Health Education, PRIME, and Strategic Consultants Intl.; and royalties for out-licensed monoclonal antibodies through UTHealth from Millipore Corporation.

Ethics approval The study was approved by central and local ethics committees and conducted in accordance with the International Council on Harmonisation Guidelines for Good Clinical Practice and the Declaration of Helsinki.

Consent to participate All patients in EVOLVE-MS-2 provided written informed consent prior to study participation.

Open Access This article is licensed under a Creative Commons Attribution-NonCommercial 4.0 International License, which permits any non-commercial use, sharing, adaptation, distribution and reproduction 
in any medium or format, as long as you give appropriate credit to the original author(s) and the source, provide a link to the Creative Commons licence, and indicate if changes were made. The images or other third party material in this article are included in the article's Creative Commons licence, unless indicated otherwise in a credit line to the material. If material is not included in the article's Creative Commons licence and your intended use is not permitted by statutory regulation or exceeds the permitted use, you will need to obtain permission directly from the copyright holder.To view a copy of this licence, visit http://creativecommons.org/licenses/by-nc/4.0/.

\section{References}

1. Biogen. Vumerity ${ }^{\circledR}$ (diroximel fumarate) prescribing information and patient information. 2019. https://www.vumerity.com/conte nt/dam/commercial/vumerity/pat/en_us/pdf/vumerity-prescribin g-information.pdf. Accessed 19 Dec 2019.

2. Biogen. Tecfidera ${ }^{\circledR}$ (dimethyl fumarate) prescribing information and patient information. 2019. https://www.tecfidera.com/conte nt/dam/commercial/multiple-sclerosis/tecfidera/pat/en_us/pdf/ full-prescribing-info.pdf. Accessed 9 Apr 2019.

3. Wehr A, Hard M, Yu M, Leigh-Pemberton R, von Moltke L. Relative bioavailability of monomethyl fumarate after administration of ALKS 8700 and dimethyl fumarate in healthy subjects. Neurology. 2018;90(Suppl. 15):P1.403.

4. Gold R, Kappos L, Arnold DL, Bar-Or A, Giovannoni G, Selmaj K, DEFINE Study Investigators, et al. Placebo-controlled phase 3 study of oral BG-12 for relapsing multiple sclerosis. N Engl J Med. 2012;367(12):1098-107.

5. Fox RJ, Miller DH, Phillips JT, Hutchinson M, Havrdova E, Kita M, CONFIRM Study Investigators, et al. Placebo-controlled phase 3 study of oral BG-12 or glatiramer in multiple sclerosis. N Engl J Med. 2012;367(12):1087-97.

6. Gold R, Arnold DL, Bar-Or A, Hutchinson M, Kappos L, Havrdova $\mathrm{E}$, et al. Long-term effects of delayed-release dimethyl fumarate in multiple sclerosis: interim analysis of ENDORSE, a randomized extension study. Mult Scler. 2017;23(2):253-65.

7. Cohan SL, Moses H, Calkwood J, Tornatore C, LaGanke C, Smoot $\mathrm{KE}$, et al. Clinical outcomes in patients with relapsing-remitting multiple sclerosis who switch from natalizumab to delayed-release dimethyl fumarate: a multicenter retrospective observational study (STRATEGY). Mult Scler Relat Disord. 2018;22:27-34.

8. Kresa-Reahl K, Repovic P, Robertson D, Okwuokenye M, Meltzer L, Mendoza JP. Effectiveness of delayed-release dimethyl fumarate on clinical and patient-reported outcomes in patients with relapsing multiple sclerosis switching from glatiramer acetate: RESPOND, a prospective observational study. Clin Ther. 2018;40(12):2077-87.

9. Fox EJ, Vasquez A, Grainger W, Ma TS, von Hehn C, Walsh $\mathrm{J}$, et al. Gastrointestinal tolerability of delayed-release dimethyl fumarate in a multicenter, open-label study of patients with relapsing forms of multiple sclerosis (MANAGE). Int J MS Care. 2016;18(1):9-18.

10. Phillips JT, Selmaj K, Gold R, Fox RJ, Havrdova E, Giovannoni $\mathrm{G}$, et al. Clinical significance of gastrointestinal and flushing events in patients with multiple sclerosis treated with delayedrelease dimethyl fumarate. Int J MS Care. 2015;17(5):236-43.

11. Min J, Cohan S, Alvarez E, Sloane J, Phillips JT, van der Walt A, et al. Real-world characterization of dimethyl fumarate-related gastrointestinal events in multiple sclerosis: management strategies to improve persistence on treatment and patient outcomes. Neurol Ther. 2019;8(1):109-19.

12. Vollmer B, Nair KV, Sillau SH, Corboy J, Vollmer T, Alvarez E. Comparison of fingolimod and dimethyl fumarate in the treatment of multiple sclerosis: two-year experience. Mult Scler J Exp Transl Clin. 2017;3(3):2055217317725102.

13. Palte MJ, Wehr A, Tawa M, Perkin K, Leigh-Pemberton R, Hanna $\mathrm{J}$, et al. Improving the gastrointestinal tolerability of fumaric acid esters: early findings on gastrointestinal events with diroximel fumarate in patients with relapsing-remitting multiple sclerosis from the phase 3, open-label EVOLVE-MS-1 study. Adv Ther. 2019;36(11):3154-65.

14. Naismith R, Wolinsky J, Wundes A, LaGanke C, Arnold D, Obradovic D, et al. Diroximel fumarate (DRF) in patients with relapsing-remitting multiple sclerosis: interim safety and efficacy results from the phase 3 EVOLVE-MS-1 study. Mult Scler. 2019. https:// doi.org/10.1177/1352458519881761 (Epub ahead of print).

15. Chow S-C, Chang M. Adaptive design methods in clinical trials - a review. Orphanet J Rare Dis. 2008;3(1):11.

16. US Food and Drug Administration. Adaptive designs for clinical trials of drugs and biologics: guidance for industry. 2018. https:// www.fda.gov/media/78495/download. Accessed 26 Aug 2019.

17. Morgan CC. Sample size re-estimation in group-sequential response-adaptive clinical trials. Stat Med. 2003;22(24):3843-57.

18. Polman CH, Reingold SC, Banwell B, Clanet M, Cohen JA, Filippi M, et al. Diagnostic criteria for multiple sclerosis: 2010 revisions to the McDonald criteria. Ann Neurol. 2011;69(2):292-302.

19. Gold R, Schlegel E, Elias-Hamp B, Albert C, Schmidt S, Tackenberg B, et al. Incidence and mitigation of gastrointestinal events in patients with relapsing-remitting multiple sclerosis receiving delayed-release dimethyl fumarate: a German phase IV study (TOLERATE). Ther Adv Neurol Disord. 2018;11:1756286418768775.

20. Phillips JT, Agrella S, Fox RJ. Dimethyl fumarate: a review of efficacy and practical management strategies for common adverse events in patients with multiple sclerosis. Int J MS Care. 2017;19(2):74-83.

21. Singer B, Lucas S, Kresa-Reahl K, Perrin Ross A, Blake P. Review: optimizing adherence to multiple sclerosis therapies. Int J MS Care. 2008;10(4):113-26. 


\section{Affiliations}

Robert T. Naismith ${ }^{1}$ - Annette Wundes ${ }^{2} \cdot$ Tjalf Ziemssen $^{3}$ - Elzbieta Jasinska ${ }^{4} \cdot$ Mark S. Freedman $^{5}$. Anthony J. Lembo ${ }^{6} \cdot$ Krzysztof Selmaj $^{7,8}$. Ilda Bidollari ${ }^{9} \cdot$ Hailu Chen $^{10}$. Jerome Hanna ${ }^{11}$.

Richard Leigh-Pemberton ${ }^{9} \cdot$ Maria Lopez-Bresnahan ${ }^{9} \cdot$ Jennifer Lyons ${ }^{10} \cdot$ Catherine Miller $^{10} \cdot$ David Rezendes $^{9}$. Jerry S. Wolinsky ${ }^{12}$ on behalf of The EVOLVE-MS-2 Study Group

Catherine Miller

catherine.miller@biogen.com

1 Washington University School of Medicine, St. Louis, MO, USA

2 Department of Neurology, University of Washington Medical Center, Seattle, WA, USA

3 Center of Clinical Neuroscience, Carl Gustav Carus University Hospital, Dresden, Germany

4 Collegium Medicum UJK, and Clinical Center, RESMEDICA, Kielce, Poland

5 University of Ottawa and the Ottawa Hospital Research Institute, Ottawa, ON, Canada
6 Division of Gastroenterology, Department of Medicine, Beth Israel Deaconess Medical Center, Harvard Medical School, Boston, MA, USA

7 Center of Neurology, Lodz, Poland

8 Department of Neurology, University of Warmia and Mazury, Olsztyn, Poland

9 Alkermes Inc., Waltham, MA, USA

10 Biogen, 225 Binney St, Cambridge, MA 02142, USA

11 Biogen, Maidenhead, UK

12 Department of Neurology, McGovern Medical School, University of Texas Health Science Center at Houston (UTHealth), Houston, TX, USA 